\title{
Using assignment examples to infer weights for ELECTRE TRI method: Some experimental results
}

\author{
V. Mousseau ${ }^{\mathrm{a}, *}$, J. Figueira ${ }^{\mathrm{a}, \mathrm{b}, 1}, \mathrm{~J}^{-}-\mathrm{Ph}$. Naux ${ }^{\mathrm{a}}$ \\ ${ }^{a}$ LAMSADE, Université Paris-Dauphine, Place du Maréchal De Lattre de Tassigny, 75775, Paris Cedex 16, France \\ ${ }^{\mathrm{b}}$ School of Economics, University of Coimbra, Av. Dias da Silva 165, 3004-512 Coimbra, Portugal
}

Received 3 December 1997; accepted 15 February 1999

\begin{abstract}
Given a finite set of alternatives $A$, the sorting (or assignment) problem consists in the assignment of each alternative to one of the pre-defined categories. In this paper, we are interested in multiple criteria sorting problems and, more precisely, in the existing method ELECTRE TRI. This method requires the elicitation of preferential parameters (weights, thresholds, category limits,...) in order to construct a preference model which the decision maker (DM) accepts as a working hypothesis in the decision aid study. A direct elicitation of these parameters requiring a high cognitive effort from the DM (V. Mosseau, R. Slowinski, Journal of Global Optimization 12 (2) (1998) 174), proposed an interactive aggregation-disaggregation approach that infers ELECTRE TRI parameters indirectly from holistic information, i.e., assignment examples. In this approach, the determination of ELECTRE TRI parameters that best restore the assignment examples is formulated through a nonlinear optimization program.

In this paper, we consider the subproblem of the determination of the weights only (the thresholds and category limits being fixed). This subproblem leads to solve a linear program (rather than nonlinear in the global inference model). Numerical experiments were conducted so as to check the behaviour of this disaggregation tool. Results showed that this tool is able to infer weights that restores in a stable way the assignment examples and that it is able to identify "inconsistencies" in the assignment examples. (c) 2001 Elsevier Science B.V. All rights reserved.
\end{abstract}

Keywords: Sorting problematic; Preference disaggregation; Weight elicitation; Numerical experiments

\footnotetext{
${ }^{*}$ Corresponding author. Tel.: +33-1-44-05-44-01; fax: $+33-1-$ 44-05-40-91.

E-mail addresses: mousseau@lamsade.dauphine.fr (V. Mousseau), figueira@lamsade.dauphine.fr (J. Figueira).

${ }^{1}$ Tel.: +351-239-790-590; fax: +351-239-40-35-11.
}

\section{Introduction}

When modeling a real world decision problem, one can face situations in which the decision can be formulated in terms of the assignment of a set of potential alternatives $A=\left\{a_{1}, a_{2}, \ldots, a_{l}\right\}$ to one of pre-defined categories. The assignment of an alternative $a$ to the appropriate category should rely on the intrinsic value of $a$ (and not 
on the comparison of $a$ to other alternatives from $A$ ).

In this paper, we are interested in the multiple criteria sorting problematic and, more precisely, in an existing multiple criteria method called ELECTRE TRI (see $[12,13,18,26]$ ). When using this method, the analyst must determine values of several parameters (profiles that define the limits between the categories, weights, discrimination thresholds, ...). The set $\pi$ of these parameters is used to construct a preference model with which the decision maker (DM) accept as a working hypothesis in the decision aid study. Apart from some very specific cases, it is not realistic to assume that the DM would be able to give explicitly the values of each parameter in $\pi$. They are far different from the natural terms in which the DM usually expresses his/her preferences and expertise.

A realistic approach consists in infering the model parameters of ELECTRE TRI through an analysis of assignment examples given by the DM, i.e., from holistic information on his/her judgments. This approach aims at substituting assignment examples for direct elicitation of the model parameters. The values of the parameters will be inferred through a certain kind of regression on assignment examples.

Mousseau and Slowinski [11] proposed an approach that infer all ELECTRE TRI parameters simultaneously starting from assignment examples. In this approach, the determination of the parameter's values that best fit the assignment examples given by a DM stems from the resolution of a nonlinear mathematical program. This optimization procedure is integrated in an interactive tool that enables the DM (or anyone acting on his/ her account) to react on the set of obtained parameters and to get insights on his/her preferences.

Although Mousseau and Slowinski [11] proposed to infer simultaneously weights, profiles and thresholds, we consider in this paper the problem of the inference of the weight vector only (in this particular case the mathematical program to be solved becomes linear). Our paper presents numerical results obtained in a laboratory experiment aiming at validating the practical usefulness of the weight inference procedure in an interactive process (preliminary results may be found in [14]).
The experimental questions are as follows (they are operationalized through the experimental design described in Section 5):

- Let $w^{\text {opt }}$ be the weight vector obtained using the linear optimization procedure on the basis of the assignment to categories of alternatives from a set $A^{*} \subset A$. Let $w^{\mathrm{dm}}$ be a weight vector expressed by the DM or inferred by the analyst from DM's assertions. Are the assignments of alternatives from $A^{*}$ more "stable" when using $w^{\text {opt }}$ than when considering $w^{\mathrm{dm}}$ (the term stable is used as insensitive of the assignments to changes of the weight vector). In other words, is the tool able to increase the "stability" of assignments of alternatives in a set $A^{*}$ ?

- The obtained weight vector $w^{\text {opt }}$ depend on the information given as input, i.e., on the set of assignment examples. What is the average amount of information necessary to "calibrate" the model in a satisfactory way? How large should $A^{*}$ be in order to derive $w^{\text {opt }}$ in a reliable manner?

- In practical decision situations, real DMs do not always provide reliable information. Due to time constraints and cognitive limitations, DMs express contradictory information, their preferences change over time ... The optimization procedure should be able to highlight the assignment examples that are contradictory or not representable through the ELECTRE TRI preference model. This experiment aims at investigating the ability of the tool to "identify" the inconsistencies in the DM's statements in order to help him/her in revising the preference information. How reliable is the optimization procedure to identify inconsistencies in the DM's judgments?

- The output of the optimization phase rely on the choice of an objective function. As different objective functions can be considered, it is important to check the variability of the output to the different functions. Does the choice of a specific objective function strongly impact the results?

The paper is organized as follows. In the next section, we present the general approach used by the inference tool. A brief description of the ELECTRE TRI method is given in Section 3. 
Section 4 describes the optimization procedure and Section 5 describes the experimental design. Section 6 presents the results and Section 7 presents the conclusions.

\section{General scheme of the approach}

The general scheme of our inference procedure is presented in Fig. 1. Its aim is to find an ELECTRE TRI model as compatible as possible with the assignment examples given by the user (the user being either the DM himself/herself or anyone acting on his/her account). The assignment examples concern a subset $A^{*} \subset A$ of alternatives for which the user has clear preferences, i.e., alternatives that the user can easily assign to a category, taking into account their evaluation on all criteria. The compatibility between the ELECTRE TRI model and the assignment examples is understood as an ability of the ELECTRE TRI method using this model to reassign the alternatives from $A^{*}$ in the same way as the user did.

In order to minimize the differences between the assignments made by ELECTRE TRI and the assignments made by the user, an optimization procedure is used. The resulting ELECTRE TRI model is denoted by $M_{\pi}$. The user can tune the model in the course of an interactive procedure. $\mathrm{He} / \mathrm{she}$ may either revise the assignment examples or fix values (or intervals of variation) for some model parameters. In the former case, the user may:

- remove and/or add some alternatives from/to $A^{*}$,

- change the assignment of some alternatives from $A^{*}$.

In the latter case, the user can give additional information on the range of variation of some model parameters basing on his/her own intuition. For example, he/she may specify:

- ordinal information on the importance of criteria,

- noticeable differences on the scales of criteria,

- incomplete definition of some profiles defining the limits between categories.

When the model is not perfectly compatible with the assignment examples, the procedure should be able to detect all "hard cases", i.e., the alternatives for which the assignment computed by the model strongly differs from the user's assignment. The user could then be asked to reconsider his/her judgment.

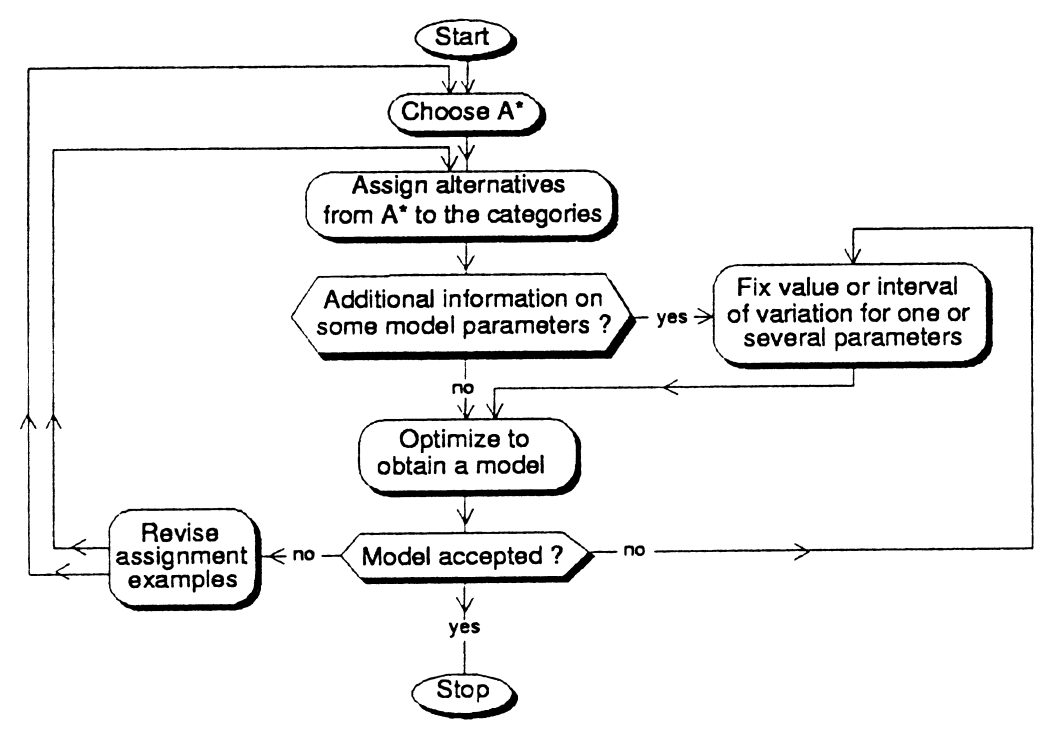

Fig. 1. General scheme of the inference procedure. 
Inferring a form of knowledge from examples of expert's decisions is a typical approach of artificial intelligence. Induction of rules or decision trees from examples in machine learning (see $[8,17])$, knowledge acquisition based on rough sets (see $[2,16,24])$, supervised learning of neural nets $($ see $[1,25])$ are well-known representatives of this approach. The appeal of this approach is that the experts are typically more confident exercising their decisions than explaining them.

In Multiple Criteria Decision Analysis, this approach is concordant with the principle of posterior rationality (see [6]) and with the aggregation-disaggregation logic used for the construction of a preference model in UTA-like procedures (see $[3-5,15,23])$. It has been also applied for the elicitation of weights used for the construction of an outranking relation in the DIVAPIME method (see $[9,10])$.

\section{Presentation of the ELECTRE TRI method}

ELECTRE TRI is a multiple criteria assignment method, i.e., a method that assigns alternatives to predefined ordered categories. The limit between two consecutive categories is formalized by what we call a profile (see Fig. 2). The assignment of an alternative $a$ results from the comparison of $a$ with the profiles defining the limits of the categories. Let $F$ denote the set of the indices of the criteria $g_{1}, g_{2}, \ldots, g_{m}(F=\{1,2, \ldots, m\})$ and $B$ the set of indices of the profiles defining $p+1$ categories $(B=\{1,2, \ldots, p\}), b_{h}$ being the upper limit of category $C_{h}$ and the lower limit of category $C_{h+1}, h=1,2, \ldots, p$ (see Fig. 2). In what follows, we will assume, without any loss of generality, that preferences increase with the value on each criterion.

ELECTRE TRI uses an outranking relation $S$ (see [20]), i.e., validates or invalidates the assertion $a S b_{h}$ (and $b_{h} S a$ ), whose meaning is " $a$ is at least as good as $b_{h}$ ". Preferences restricted to the significance axis of each criterion are defined through pseudo-criteria (see [21,22] for details on this double-threshold preference representation). The indifference and preference thresholds, $q_{j}\left(b_{h}\right)$ and $p_{j}\left(b_{h}\right)$, constitute the intra-criterion preferential information.

They account for the imprecise nature of the evaluations $g_{j}(a)$ (see [19]). The indifference threshold $q_{j}\left(b_{h}\right)$ specifies the largest difference $g_{j}(a)-g_{j}\left(b_{h}\right)$ they preserves indifference between $a$ and $b_{h}$ on criterion $g_{j}$ while the preference threshold $p_{j}\left(b_{h}\right)$ represents the smallest difference $g_{j}(a)-g_{j}\left(b_{h}\right)$ compatible with $a$ preference in favor of $a$ on criterion $g_{j}$.

At the comprehensive level of preferences, in order to validate the assertion $a S b_{h}$ (or $b_{h} S a$ ), two conditions should be verified.

- concordance: for an outranking $a S b_{h}$ (or $b_{h} S a$ ) to be accepted, a "sufficient" majority of criteria should be in favor of this assertion,

- non-discordance: when the concordance condition holds, none of the criteria in the minority

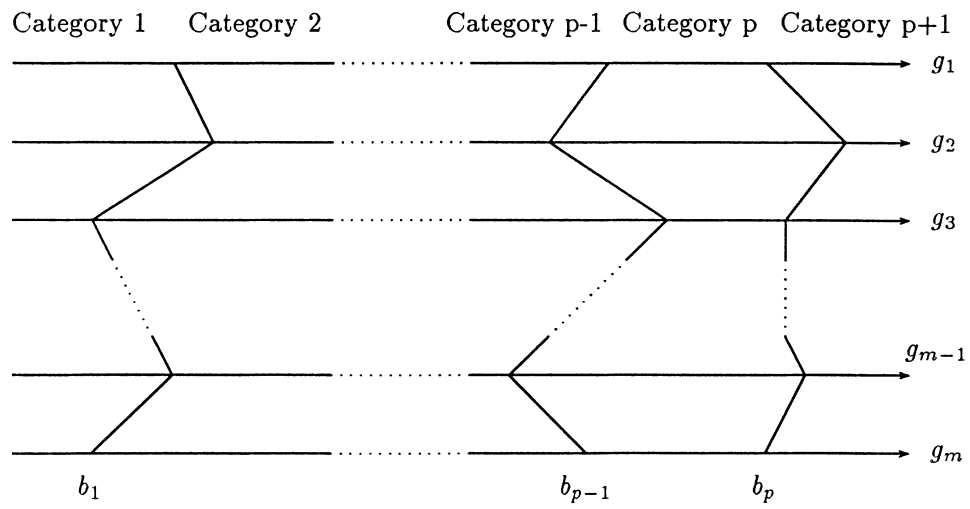

Fig. 2. Definition of categories using limit profiles. 
should oppose to the assertion $a S b_{h}$ (or $b_{h} S a$ ) in a "too strong way".

Two types of inter-criteria preference parameters intervene in the construction of $S$ :

- the set of weight-importance coefficients $\left(w_{1}, w_{2}, \ldots, w_{m}\right)$ is used in the concordance test when computing the relative importance of the coalitions of criteria being in favor of the assertion $a S b_{h}$,

- the set of veto thresholds $\left(v_{1}\left(b_{h}\right), \ldots\right.$, $\left.v_{j}\left(b_{h}\right), \ldots, v_{m}\left(b_{h}\right)\right)$ is used in the discordance test; $v_{j}\left(b_{h}\right)$ represents the smallest difference $g_{j}\left(b_{h}\right)-g_{j}(a)$ incompatible with the assertion $a S b_{h}$.

ELECTRE TRI builds an index $\sigma\left(a, b_{h}\right) \in[0,1]$ $\left(\sigma\left(b_{h}, a\right)\right.$, resp. $)$ that represents the degree of credibility of the assertion $a S b_{h} \quad\left(b_{h} S_{a}\right.$, resp.), $\forall a \in A, \forall h \in B$. The assertion $a S b_{h}\left(b_{h} S a\right.$, resp.) is considered to be valid if $\sigma\left(a, b_{h}\right) \geqslant \lambda\left(\sigma\left(b_{h}, a\right) \geqslant \lambda\right.$, resp.), $\lambda$ being a "cutting level" such that $\lambda \in[0.5,1]$ (see [18] for a justification of the construction of this index).

Determining $\sigma\left(a, b_{h}\right)$ consists of the following steps (the value of $\sigma\left(b_{h}, a\right)$ is computed analogously):

1. compute the partial concordance indices $c_{j}\left(a, b_{h}\right) \forall j \in F$

$$
c_{j}\left(a, b_{h}\right)=\left\{\begin{array}{c}
0 \quad \text { if } g_{j}\left(b_{h}\right)-g_{j}(a) \geqslant p_{j}\left(b_{h}\right), \\
1 \text { if } g_{j}\left(b_{h}\right)-g_{j}(a) \leqslant q_{j}\left(b_{h}\right), \\
\frac{p_{j}\left(b_{h}\right)+g_{j}(a)-g_{j}\left(b_{h}\right)}{p_{j}\left(b_{h}\right)-q_{j}\left(b_{h}\right)} \quad \text { otherwise, }
\end{array}\right.
$$

2. compute the comprehensive concordance index $c\left(a, b_{h}\right)$

$$
c\left(a, b_{h}\right)=\frac{\sum_{j \in F} w_{j} c_{j}\left(a, b_{h}\right)}{\sum_{j \in F} w_{j}},
$$

3. compute the discordance indices $d_{j}\left(a, b_{h}\right)$ $\forall j \in F$

$$
d_{j}\left(a, b_{h}\right)=\left\{\begin{array}{cc}
0 \quad \text { if } g_{j}\left(b_{h}\right)-g_{j}(a) \leqslant p_{j}\left(b_{h}\right), \\
1 \quad \text { if } g_{j}\left(b_{h}\right)-g_{j}(a)>v_{j}\left(b_{h}\right), \\
\frac{g_{j}\left(b_{h}\right)-g_{j}(a)-p_{j}\left(b_{h}\right)}{v_{j}\left(b_{h}\right)-p_{j}\left(b_{h}\right)} \quad \text { otherwise, }
\end{array}\right.
$$

4. compute the credibility index $\sigma\left(a, b_{h}\right)$ of the outranking relation

$$
\sigma\left(a, b_{h}\right)=c\left(a, b_{h}\right) \prod_{j \in \bar{F}} \frac{1-d_{j}\left(a, b_{h}\right)}{1-c\left(a, b_{h}\right)},
$$

where

$$
\bar{F}=\left\{j \in F: d_{j}\left(a, b_{h}\right)>c\left(a, b_{h}\right)\right\}
$$

The values of $\sigma\left(a, b_{h}\right), \sigma\left(b_{h}, a\right)$ and $\lambda$ determine the preference situation between $a$ and $b_{h}$ :

- $\sigma\left(a, b_{h}\right) \geqslant \lambda$ and $\sigma\left(b_{h}, a\right) \geqslant \lambda \Rightarrow a S b_{h}$ and $b_{h} S a \Rightarrow a I b_{h}$, i.e., $a$ is indifferent to $b_{h}$,

- $\sigma\left(a, b_{h}\right) \geqslant \lambda$ and $\sigma\left(b_{h}, a\right)<\lambda \Rightarrow a S b_{h}$ and not $b_{h} S a \Rightarrow a \succ b_{h}$, i.e., $a$ is preferred to $b_{h}$ (weakly or strongly),

- $\sigma\left(a, b_{h}\right)<\lambda$ and $\sigma\left(b_{h}, a\right) \geqslant \lambda \Rightarrow$ not $a S b_{h}$ and $b_{h} S a \Rightarrow b_{h} \succ a$, i.e., $b_{h}$ is preferred to $a$ (weakly or strongly),

- $\sigma\left(a, b_{h}\right)<\lambda$ and $\sigma\left(b_{h}, a\right)<\lambda \Rightarrow$ not $a S b_{h}$ and not $b_{h} S a \Rightarrow a R b_{h}$, i.e., $a$ is incomparable to $b_{h}$.

Two assignment procedures are then available (the role of these exploitation procedures is then to analyse the way in which an alternative $a$ compares to the profiles so as to determine the category to which $a$ should be assigned).

Pessimistic (or conjunctive) procedure:

(a) compare $a$ successively to $b_{i}$, for $i=$ $p, p-1, \ldots, 1$,

(b) $b_{h}$ being the first profile such that $a S b_{h}$, assign $a$ to category $C_{h+1}\left(a \rightarrow C_{h+1}\right)$.

Optimistic (or disjunctive) procedure:

(a) compare $a$ successively to $b_{i}, i=1,2, \ldots, p$,

(b) $b_{h}$ being the first profile such that $b_{h} \succ a$, assign $a$ to category $C_{h}\left(a \rightarrow C_{h}\right)$.

If $b_{h-1}$ and $b_{h}$ denote the lower and upper profile of the category $C_{h}$, the pessimistic (or conjunctive) procedure assigns alternative $a$ to the highest category $C_{h}$ such that $a$ outranks $b_{h-1}$, i.e., $a S b_{h-1}$. When using this procedure with $\lambda=1$, an alternative $a$ can be assigned to category $C_{h}$ only if $g_{j}(a)$ equals or exceeds $g_{j}\left(b_{h}\right)$ (up to a threshold) for each criterion (conjunctive rule).

The optimistic (or disjunctive) procedure assigns $a$ to the lowest category $C_{h}$ for which the lower profile $b_{h}$ is preferred to $a$, i.e., $b_{h} \succ a$. When using this procedure with $\lambda=1$, an alternative $a$ can be assigned to category $C_{h}$ when $g_{j}\left(b_{h}\right)$ exceeds $g_{j}(a)$ (up to a threshold) at least for one criterion 
(disjunctive rule). When $\lambda$ decreases, the conjunctive and disjunctive characters of these rules are weakened.

\section{The optimization procedure}

The set $\pi$ of parameters of an ELECTRE TRI model are:

- the profiles defined by their evaluations $g_{j}\left(b_{h}\right) \forall j \in F, \forall h \in B$,

- the importance coefficients $w_{j} \forall j \in F$,

- the indifference and preference thresholds $q_{j}\left(b_{h}\right), p_{j}\left(b_{h}\right) \forall j \in F, \forall h \in B$,

- the veto thresholds $v_{j}\left(b_{h}\right) \forall j 2 \in F, \forall h \in B$.

The conducted experiment considered the case where the profiles and thresholds are known and where the weights are to be inferred. Moreover, we will confine our analysis to the case where the pessimistic assignment procedure is used.

\subsection{Variables of the problem}

In ELECTRE TRI pessimistic assignment procedure, an alternative $a_{k}$ is assigned to category $C_{h}\left(b_{h-1}\right.$ and $b_{h}$ being the lower and upper profiles of $C_{h}$, respectively) iff $\sigma_{\pi}\left(a_{k}, b_{h-1}\right) \geqslant \lambda$ and $\sigma_{\pi}\left(a_{k}, b_{h}\right)<\lambda$ (where $\sigma_{\pi}$ is the credibility index related to the set of parameters $\pi$ ).

Let us suppose that the DM has assigned the alternative $a_{k} \in A^{*}$ category $C_{h k}\left(a_{k} \rightarrow C_{h k}\right)$. Let us define the slack variables $x_{k}$ and $y_{k}$ such that $\sigma_{\pi}\left(a_{k}, b_{h k-1}\right)-x_{k}=\lambda$ and $\sigma_{\pi}\left(a_{k}, b_{h_{k}}\right)+y_{k}=\lambda$.

The optimization problem will include the following variables:

$x_{k}, y_{k} \forall k$ such that $a_{k} \in A^{*}$

$\lambda$

$w_{j} \forall j \in F$ slack variables $(2 n)$ cutting level (1) importance coefficients $(m)$

\subsection{An accuracy criterion}

If the values of the slack variables $x_{k}$ and $y_{k}$ are both positive then ELECTRE TRI pessimistic assignment procedure will assign alternative $a_{k}$ to

the "correct" category. If, however, one or both of these values are negative, the ELECTRE TRI pessimistic assignment procedure will assign alternative $a_{k}$ to a "wrong" category. The lower the minimum of these two values, the less adapted is the model $M_{\pi}$ to give an account of the assignment of $a_{k}$ made by the DM. Moreover, if $x_{k}$ and $y_{k}$ are both positive then $a_{k}$ is assigned consistently with the DM's statement for all $\lambda^{\prime} \in\left[\lambda-y+k, \lambda+x_{k}\right]$.

Let us consider now the set of alternatives $A^{*} \subset A$ where $\operatorname{card}\left(A^{*}\right)=n$ and suppose that the DM has assigned the alternative $a_{k}$ to the category $C_{h_{k}} \forall a_{k} \in A^{*}$. The model $M_{\pi}$ will be consistent with DM's assignments iff $x_{k} \geqslant 0$ and $y_{k} \geqslant 0 \forall k$ such that $a_{k} \in A^{*}$.

Consistently with the preceding argument, an accuracy criterion can be defined as

$\max _{k: a_{k} \in A^{*}}\left\{\min \left\{x_{k}, y_{k}\right\}\right\}$.

We obtain a standard MaxMin problem. If the accuracy criterion takes a non-negative value then all alternatives contained in $A^{*}$ are "correctly" assigned for all

$\lambda^{\prime} \in\left[\lambda-\min _{k: a_{k} \in A^{*}}\left\{y_{k}\right\}, \lambda+\min _{k: a_{k} \in A^{*}}\left\{x_{k}\right\}\right]$.

This criterion, however, takes into account the "worst case" only, i.e., the alternative for which the ELECTRE TRI model gives the most different assignment from the DM. An accuracy criterion should be able to take into account an average information concerning the accuracy of the model, i.e., its overall ability to assign the alternatives from $A^{*}$ to the correct category. Hence, we propose to replace criterion (5) by the following one:

$\max _{k: a_{k} \in A^{*}}\left\{\min \left\{x_{k}, y_{k}\right\}+\varepsilon \sum_{k: a_{k} \in A^{*}}\left(x_{k}+y_{k}\right)\right\}$,

where $\varepsilon$ is a small positive value. Eq. (6) can be rewritten as

$$
\begin{array}{ll}
\max & \left\{\alpha+\varepsilon \sum_{k: a_{k} \in A^{*}}\left(x_{k}+y_{k}\right)\right\}, \\
\text { s.t. } & \alpha \leqslant x_{k} \quad \forall k \text { such that } a_{k} \in A^{*}, \\
& \alpha \leqslant y_{k} \quad \forall k \text { such that } a_{k} \in A^{*} .
\end{array}
$$




\subsection{Constraints of the problem}

The constraints of the optimization problem are the following:

$$
\begin{aligned}
& \sigma_{\pi}\left(a_{k}, b_{h_{k-1}}-x_{k}=\lambda\right. \\
& \forall k: a_{k} \in A^{*} \\
& \sigma_{\pi}\left(a_{k}, b_{h_{k}}\right)+y_{k}=\lambda \\
& \forall k: a_{k} \in A^{*} \\
& \alpha \leqslant x_{k}, \alpha \leqslant y_{k} \forall k: \\
& a_{k} \in A^{*} \\
& \lambda \in[0.5,1] \\
& w_{j} \geqslant 0 \forall j \in F
\end{aligned}
$$

definition of the slack variables $x_{k}(n)$ definition of the slack variables $y_{k}(n)$ definition of $\alpha(2 n)$ interval of variation for $\lambda$ (2) non-negativity constraints $(m)$

Additional constraints can be added in the course of the interactive procedure in order to take into account an intuitive view of the DM on the value of some parameters. For instance, if the DM does not consider any criterion as a dictator, an appropriate constraint is $w_{j} \leqslant(1 / 2) \sum_{i=1}^{m}$ $w_{i} \forall j \in F$.

\subsection{Optimization problem to be solved}

The basic form of the optimization problem to be solved is the following:

$$
\max \left\{\alpha+\varepsilon \sum_{k: a_{k} \in A^{*}}\left(x_{k}+y_{k}\right)\right\}
$$

s.t.

$\alpha \leqslant x_{k} \quad \forall k$ such that $a_{k} \in A^{*}$,

$\alpha \leqslant y_{k} \quad \forall k$ such that $a_{k} \in A^{*}$,

$\sum_{j=1}^{m} w_{j} c_{j}\left(a_{k}, b_{h_{k}-1}\right)-x_{k}=\lambda$

$\forall k$ such that $a_{k} \in A^{*}$,

$\sum_{j=1}^{m} w_{j} c_{j}\left(a_{k}, b_{h_{k}}\right)+y_{k}=\lambda$

$\forall k$ such that $a_{k} \in A^{*}$,

$\sum_{j=1}^{m} w_{j}=1$,

$\lambda \in[0.5,1]$,

$w_{j} \geqslant 0, \quad \forall j \in F$.
As the objective function and all constraints are linear, the above problem is a linear programming problem. It contains $2 n+m+1$ variables and $4 n+m+2$ constraints. Let us remark that the slack variables $x_{k}$ and $y_{k}$ can be eliminated from the problem formulation since they are defined by the constraints (13) and (14). This elimination reduces the number of variables.

\section{Experimental design}

This experiment is a laboratory work, i.e., takes its material in a past real world case study to perform a posteriori computations in order to test the operational validity of the optimization model proposed in Section 4. The data considered comes from the real world application described in $[26,27]$.

This application considers the problem of assigning a set $A$ of 100 alternatives $\left(A=\left\{a_{1}\right.\right.$, $\left.a_{2}, \ldots, a_{100}\right\}$ is described in [7]) to three (the initial data specified 5 categories; we grouped the three top categories $\left(C_{3}, C_{4}\right.$ and $\left.C_{5}\right)$ as none of the alternatives were assigned by the ELECTRE TRI model to $C_{4}$ and $C_{5}$ ) ordered categories $C_{1}, C_{2}$ and $C_{3}$ (two limit profiles $b_{1}$ and $b_{2}$ define the "frontiers" $C_{1}-C_{2}$ and $C_{2}-C_{3}$ ) on the basis of 7 criteria (preferences on all criteria are decreasing with the evaluations, i.e., the lower the better).

As no interaction with the DM is possible, we consider the assignment of ELECTRE TRI pessimistic assignment procedure (with the parameters given in [26]) as assignment examples expressed by a "fictitious" DM. The experimental method consist in using the optimization procedure with different subsets of assignment examples to infer the weights that "best" match with the examples (with the given values for profiles and thresholds).

So as to get consistent results, we generate 80 subsets of $A$, the cardinality of these subset being either $6,12,18,24,30,36,42$ or 48 (10 sets of each size were generated). Each of these subsets is conceived so that the alternatives are assigned uniformly on the three categories. Let us denote $A_{i}^{j}$ the $j$ th set of size $i$. In order to test the ability of the optimization procedure to identify inconsistent information (see Section 6.3), we consider $\operatorname{Err}_{i}^{j}$ 
Table 1

Type of errors introduced in the sets

\begin{tabular}{lll}
\hline $\begin{array}{l}\text { Initial } \\
\text { category }\end{array}$ & $\begin{array}{l}\text { Error } \\
\text { category }\end{array}$ & $\begin{array}{l}\text { Number in each } \\
\text { sample }\end{array}$ \\
\hline$C_{1}$ & $C_{2}$ & 2 \\
$C_{2}$ & $C_{1}$ & 2 \\
$C_{2}$ & $C_{3}$ & 2 \\
$C_{3}$ & $C_{2}$ & 2 \\
$C_{1}$ & $C_{3}$ & 1 \\
$C_{3}$ & $C_{1}$ & 1 \\
\hline
\end{tabular}

derived from $A_{i}^{j}$ in which an alternative is voluntarily assigned to a "wrong" category. The error introduced consist in changing the assignment of an alternative (for example, assigning to $C_{1}$ an alternative that should be assigned to $C_{2}$ ). Different types of errors were considered as shown in the Table 1. The sets of assignment examples for which computations were performed are described in [7].

The mathematical program corresponding to each set $\operatorname{Err}_{i}^{j}$ and $A_{i}^{j}$ has been solved with different objective functions (see Section 4.2). The general form of the considered objective function $z$ to be maximized is

$z=\min _{k: a_{k} \in A^{*}}\left\{x_{k}, y_{k}\right\}+\varepsilon \sum_{k: a_{k} \in A^{*}}\left(x_{k}+y_{k}\right)$

and computations have been performed for $\varepsilon=10^{-3}, 10^{-2}, 10^{-1}, 1,10^{1}, 10^{2}$. This allows us to check for the variability of the output to the choice of an objective function.

\section{Results}

The computations have been performed using Cplex on a Sun Sparc 5 workstation with $32 \mathrm{MB}$ memory. Considering the small size of the problem, the computing time never exceeded 0.1 seconds.

Prior to stating the results, it is important to mention that these results are dependent on the data under consideration. The proposed general implications should be understood taking into account this restriction. The reader will find detailed numerical results in [7].

\subsection{Is the tool able to increase the "stability" of assignments of alternatives?}

Let $w^{\text {opt }}$ be the weight vector obtained using the optimization procedure on the basis of the assignment to categories of alternatives from a set $A_{i}^{j}$. Let $w^{\mathrm{dm}}$ be the weight vector used to generate the assignment examples. A first validation of the usefulness of the optimization procedure is to check if the assignments of alternatives from $A_{i}^{j}$ are more "stable" when using $w^{\text {opt }}$ than when considering $w^{\mathrm{dm}}$, i.e., is the tool able to increase the "stability" of assignments of alternatives in a set $A_{i}^{j}$ ?

So as to answer this question, we will use the following methodology. In the mathematical program to be solved, the variable $\alpha$ is introduced to transform a MaxMin objective into a Max objective and represents the minimum value among the slack variables $x_{k}$ and $y_{k}$ (Section 4.2). The larger the $\alpha$, the more stable are the assignments of alternatives in $A_{i}^{j}$. The assignments are said to be stable if they are not affected by a modification of the cutting level $\lambda$ (or of the weights).

Let us denote $\alpha_{\mathrm{dm}}\left(A_{i}^{j}\right)$ the maximum variation on the cutting level $\lambda$ preserving correct assignment of alternatives from $A_{i}^{j}$ with the initial weights, i.e., those given in [26]. Let us denote $\alpha_{\text {opt }}\left(A_{i}^{j}\right)$ the maximum variation on the cutting level $\lambda$ preserving correct assignment of alternatives from $A_{i}^{j}$ with the weights obtained using the optimization procedure. The improvement of the stability of the assignments provided by the procedure can be evaluated by $\alpha_{\mathrm{opt}}\left(A_{i}^{j}\right)-\alpha_{\mathrm{dm}}\left(A_{i}^{j}\right)$. Table 2 gives the numerical results.

Considering these results, we can observe:

- Firstly the results show that the larger the set of assignment examples, the less stable the assignments, i.e., the more sensitive are these assignments to a change in weights. This is a straightforward evidence as each assignment example adds two constraints to the program to be solved (see Section 4.3).

- Secondly, these results show a significant improvement of the stability of the assignments whatever the size of the set of examples (mean value: $0: 15$ ). This proves the ability of the optimization procedure to perform "good" weights 
Table 2

Improvement of the "stability" of assignments

\begin{tabular}{clll}
\hline Size: $i$ & $\bar{\alpha}_{\mathrm{opt}}(i)$ & $\bar{\alpha}_{\mathrm{dm}}(i)$ & $\bar{\alpha}_{\mathrm{opt}}(i)-\bar{\alpha}_{\mathrm{dm}}(i)$ \\
\hline 6 & 0.24 & 0.08 & 0.16 \\
12 & 0.23 & 0.02 & 0.21 \\
18 & 0.21 & 0.08 & 0.13 \\
24 & 0.20 & 0.02 & 0.18 \\
30 & 0.19 & 0.08 & 0.11 \\
36 & 0.18 & 0.03 & 0.15 \\
42 & 0.18 & 0.02 & 0.16 \\
48 & 0.10 & 0.02 & 0.08 \\
& & Mean & 0.15 \\
\hline
\end{tabular}

that enables ELECTRE TRI to reassign the alternatives in a very stable way.

\subsection{Which "amount" of information is necessary to infer the weights in a reliable way?}

In order to infer in a reliable way a weight vector $w^{\text {opt }}$, the optimization procedure requires information as input, i.e., on the set of assignment examples. What is the amount of information necessary to "calibrate" the model in a satisfactory way? How large should $A^{*}$ be in order to derive $w^{\text {opt }}$ in a reliable manner? This question is essential for practical use to the inference model in real world decision problems. The analyst should have some simple guidelines to manage the interaction with the DM avoiding unnecessary question, but collecting a sufficient information.

In order to determine a "reasonable amount of information" to infer the weights, we use the following experimental scheme: the optimization procedure is performed using different sets of assignment examples, whose size varies from 6 to 48 (10 sets for each size, see Section 5). We observe then the ability of ELECTRE TRI using the inferred weights to assign correctly the whole set of 100 alternatives. Obviously the ability of ELECTRE TRI using the inferred weights to reassign all alternatives correctly increases with the size of the set from which the weights are derived. However, the number of assignment examples expressed by the DM should not be too large.
Let us denote by $\alpha_{\mathrm{opt}}^{100}\left(A_{i}^{j}\right)$ the maximum variation on the cutting level $\lambda$ preserving correct assignment for all 100 alternatives with the weights inferred from $A_{i}^{j}$. Let $\alpha_{\mathrm{opt}}^{100}(i)$ be the mean value of the $\alpha_{\mathrm{opt}}^{100}\left(A_{i}^{j}\right)$, for all sets $A_{i}^{j}$ of size $i$.

Let us denote by $\operatorname{err}_{\mathrm{opt}}^{100}\left(A_{i}^{j}\right)$ the number of "wrong" assignments among the 100 alternatives with the weights inferred from $A_{i}^{j}$. Let $\overline{\operatorname{err}}_{\mathrm{opt}}^{100}(i)$ be the mean value of the $\operatorname{err}_{\mathrm{opt}}^{100}\left(A_{i}^{j}\right)$, for all sets $A_{i}^{j}$ of size $i$. The results of the computations are grouped in Table 3.

As foreseen, the results show that $\bar{\alpha}_{\mathrm{opt}}^{100}(i)$ increases and $\overline{\operatorname{err}}_{\mathrm{opt}}^{100}(i)$ decreases with the size $i$ of the set. Moreover, $\bar{\alpha}_{\mathrm{opt}}^{100}(i)$ becomes positive for $12<$ $i<18$; such a positive value means that weights inferred from a set of $i$ assignment examples is able (in mean value) to reassign correctly all 100 alternatives.

The number of parameters to be inferred (weights $w_{j}$ ) depend on the number of criteria only. Considering the above results, $2 \times m(m$ being the number of criteria) seems to be a reasonable number of assignment examples to infer the weights in a reliable way (as 7 criteria are considered, $12<2 m<18$ ). However, it is important to notice that using a set $A_{i}^{j}$ of $2 m$ assignment examples does not always infer weights such that $\operatorname{err}_{\mathrm{opt}}^{100}\left(A_{i}^{j}\right)=0$, i.e., some alternatives mights be incorrectly reassigned. Nevertheless, the $2 m$ seems to us a good balance between number of examples required from the DM (necessary limited) and the reliability of the inferred weights. This result needs to be reinforced by a replication of this experiment, particularly in the case where the number of categories exceeds 3 .

Table 3

Information required to infer weights reliably

\begin{tabular}{crl}
\hline Size: $i$ & $\bar{\alpha}_{\mathrm{opt}}^{100}(i)$ & $\overline{\mathrm{err}}_{\mathrm{opt}}^{100}(i)$ \\
\hline 6 & -0.093 & 4.4 \\
12 & -0.088 & 4.0 \\
18 & 0.076 & 0.6 \\
24 & 0.129 & 0.2 \\
30 & 0.157 & 0.0 \\
36 & 0.112 & 0.4 \\
42 & 0.164 & 0.0 \\
\hline
\end{tabular}


6.3. Is the tool able to identify the inconsistencies in the DM's assertions?

The optimization procedure that is tested in this experiment is conceived to be integrated in an interactive tool briefly described in Section 2. In practical decision situations, real DMs do not always provide reliable information. Due to time constraints and cognitive limitations, DM's preferences evolve over time, contains contradictory or inconsistent information. The role of an interactive tool is to help the DM to learn about his/her preferences and their possible representation in a specific aggregation model. Inconsistencies occur when the DM's preferences (in our case a set of assignment examples) can not be expressed through the preference model that is used (ELECTRE TRI in our case). In such cases, it is important to extract from the expressed preferences the inconsistent pieces of information, i.e., the most atypical or contradictory assignment examples. Consequently, a fundamental experimental issue concerns the ability of the tool to identify the inconsistencies in the DM's statements: identifying inconsistencies will help the DM in revising the expressed assertions in order for his/her preference to match the used preference model.

In order to test the ability of the optimization procedure to identify inconsistent information, we consider the sets $\operatorname{Err}_{i}^{j}$ derived from $A_{i}^{j}$ in which an alternative is voluntarily assigned to a wrong category. The error introduced consist in changing the assignment of an alternative (for example, assigning to $C_{1}$ an alternative that should be assigned to $C_{2}$ ).

So as to know if the optimization procedure is able to identify an inconsistency, we will ground on the following idea. Let $a_{k \text { err }}$ be the alternative wrongly assigned in $\operatorname{Err}_{i}^{j}, x_{k}$ err and $y_{k}$ err being the corresponding slack variables (see Section 4.1). Let us recall that the variable $\alpha$ corresponds to the minimum of $x_{k}$ and $y_{k}$ for all alternatives in $\operatorname{Err}_{i}^{j}$. The alternatives that are the "most difficult" to assign are those (in the interactive process, these alternatives are those which should be proposed to the $\mathrm{DM}$ in order to revise the assignments) for which $x_{k}=\alpha$ or $y_{k}=\alpha$. Hence, we will consider the error or inconsistency to be "discovered" if $x_{k^{\text {err }}}=\alpha$ or $y_{k^{\text {err }}}=\alpha$. If $a_{k^{\text {err }}}$ is identified as one of
Table 4

Identification of "errors"

\begin{tabular}{crrl}
\hline Size: $i$ & $\bar{\alpha}(i)$ & $\bar{n}(i)$ & $\bar{n}(i) / i(\%)$ \\
\hline 6 & 0.00 & 4.3 & 71.7 \\
12 & 0.01 & 6.9 & 57.5 \\
18 & -0.01 & 8.8 & 48.9 \\
24 & -0.01 & 8.3 & 34.6 \\
30 & 0.01 & 15.2 & 50.7 \\
36 & 0.01 & 13.0 & 36.1 \\
42 & -0.02 & 12.4 & 29.5 \\
48 & -0.02 & 14.1 & 29.4 \\
\hline
\end{tabular}

the alternative the most difficult to assign, it might not be the only alternative for which one of the slack variable equal $\alpha$. Let $n\left(\operatorname{Err}_{i}^{j}\right)$ denote the number of such alternatives; the lower $n\left(\operatorname{Err}_{i}^{j}\right)$, the more accurate is the identification. We denote $n(i)$ the mean value for $n\left(\operatorname{Err}_{i}^{j}\right)$, for all $j$.

In the result, we observe that the error is always identified $\left(x_{k^{\text {err }}}=\alpha\right.$ or $\left.y_{k_{\text {err }}}=\alpha\right)$. Table 4 gives the numerical results.

Unsurprisingly, we observe a degradation of the value of $\bar{\alpha}(i)$ compared to its value in the case of the initial assignment sets, i.e., without errors (see Table 2). Secondly, though the errors are systematically identified, the number of alternatives $\bar{n}(i)$ is increasing with the size $i$ of the sets; however, the proportion of such alternatives is decreasing with $i$. Finally the results show that the optimization procedure has a good ability to identify suspicious assignments.

\subsection{Is the output sensitive to the choice of an objective function?}

The output of the optimization phase rely on the choice of an objective function. As different objective functions can be considered, it is important to check the variability of the output to the different functions.

In this study, we investigate a class of objective functions $z(\varepsilon)$ to be maximized of the form (see Section 4.2)

$$
z(\varepsilon)=\min _{k: a_{k} \in A^{*}}\left\{x_{k}, y_{k}\right\}+\varepsilon \sum_{k: a_{k} \in A^{*}}\left(x_{k}+y_{k}\right) .
$$

These objective functions $z(\varepsilon)$ aggregate two components: 
- a first component which leads to an optimum that account only for the alternative that is the most difficult to assign correctly,

- a second additive component in which a stable assignment may be compensated by a less stable one; this second component account for the overall ability of the obtained weight vector to assign the alternatives correctly.

The parameter $\varepsilon$ enables to tune $z(\varepsilon)$ in direction of one of its two components $(\varepsilon=0$ leads to a standard MaxMin criterion while a sufficiently large value for $\alpha$ leads to an additive criterion).

In our experiment, we perform the computations for $\varepsilon=10,10^{-2}, 10^{-1}, 10^{0}, 10^{1}, 10^{2}$ and for each set $A_{i}^{j}, i=6,12,24,30,36,42,48, j=$ $1, \ldots, 10$. Firstly we can observe that different values for $\varepsilon$ leads to different results. Obviously, we observe that optimal value for $\alpha$ decreases when $\varepsilon$ increases and $\left(1 / \operatorname{card}\left(A^{*}\right)\right) \sum_{k: a_{k} \in A^{*}}\left(x_{k}+y_{k}\right)$ increases with $\varepsilon$. In other words, the stability of the worst case deteriorates while the "mean stability" increases when we emphasize the additive component of the objective function.

A more interesting point deals with the comparative reliability of the weights $w_{\text {maxmin }}$ and $w^{\text {add }}$ inferred using a pure maxmin and a standard ad- ditive objective function. More precisely, what is the ability of $w_{\operatorname{maxmin}}$ and $w^{\text {add }}$ to reassign correctly the whole set of 100 alternatives. Tables 5 and 6 presents the values of $\bar{\alpha}_{o p t}^{100}(i)$ and $\overline{\operatorname{err}}_{\mathrm{opt}}^{100}(i)$ (see Section 6.2).

We observe that:

- when the set of assignment examples is insufficiently large ( $\leqslant 12$, see Section 6.2$), w_{\text {maxmin }}$ and $w_{\text {add }}$ are equally accurate,

- when sufficient information is provided, the maxmin criterion leads to slightly more robust weights,

- the number of incorrectly reassigned alternatives are almost always equal when using $w_{\operatorname{maxmin}}$ and $w_{\text {add }}$.

While both objective functions give good results, a slight advantage is observed in favor of the maxmin criterion in terms of the stability of reassignments.

\section{Conclusions}

This paper presents an experimental validation of a procedure aiming at inferring the weights

Table 5

$\bar{\alpha}_{\mathrm{opt}}^{100}(i)$ : ability of $w$ to reassign alternatives

\begin{tabular}{llllllll}
\hline$\varepsilon$ & Size: $i$ & & & & & \\
\cline { 2 - 7 } & 6 & 12 & 18 & 24 & 30 & 36 & 42 \\
\hline 0.001 & -0.093 & -0.088 & 0.076 & 0.129 & 0.157 & 0.112 & 0.164 \\
0.01 & -0.093 & -0.088 & 0.076 & 0.129 & 0.157 & 0.112 & 0.164 \\
0.1 & -0.093 & -0.088 & 0.076 & 0.129 & 0.157 & 0.112 & 0.164 \\
1 & -0.093 & -0.088 & 0.076 & 0.129 & 0.157 & 0.112 & 0.164 \\
10 & -0.093 & -0.088 & 0.076 & 0.129 & 0.157 & 0.112 & 0.164 \\
100 & -0.093 & -0.088 & 0.076 & 0.129 & 0.157 & 0.112 & 0.164 \\
\hline
\end{tabular}

Table 6

$\overline{\operatorname{err}}_{\mathrm{opt}}^{100}(i)$ : number of incorrect reassignments

\begin{tabular}{llllllll}
\hline$\varepsilon$ & Size: $i$ & & & & & \\
\cline { 2 - 6 } & 6 & 12 & 18 & 24 & 30 & 36 & 42 \\
\hline 0.001 & 4.4 & 4.0 & 0.6 & 0.2 & 0.0 & 0.4 & 0.0 \\
0.01 & 4.4 & 4.0 & 0.6 & 0.2 & 0.0 & 0.4 & 0.0 \\
0.1 & 4.4 & 4.0 & 0.6 & 0.2 & 0.0 & 0.4 & 0.0 \\
1 & 4.4 & 4.0 & 0.6 & 0.2 & 0.0 & 0.4 & 0.0 \\
10 & 4.4 & 4.0 & 0.6 & 0.2 & 0.0 & 0.0 & 0.0 \\
100 & 4.4 & 4.0 & 0.6 & 0.2 & 0.0 & 0.4 & 0.0 \\
\hline
\end{tabular}


of the ELECTRE TRI method on the basis of assignment examples (see [11]). The performances of this procedure were tested together with its ability to be integrated in an interactive process based on the aggregation-disaggregation paradigm. In conclusion, we can state the following:

- the results show that the inference procedure derives weights that assign (using ELECTRE TRI) the examples to the correct category in a stable way,

- experimental results suggest that a "reasonable" number of assignment examples to infer the weights reliably is $2 m, m$ being the number of criteria,

- the inference procedure shows a good ability to detect inconsistencies in the user's assertions; this property is particularly important in the perspective of its integration in an interactive process,

- the different objective functions tested did not provided significantly different results in terms of reassignment performances.

Although these results depend on the data under consideration, the empirical results seems robust. These good results concerning the behaviour of the inference procedure must be analysed in relation the use of this inference procedure. The inference phase (formalized by the mathematical program) is not only a simply adjustment process, but is intended to be integrated into an interactive aggregation disaggregation process (see Section 2). This interactive process aims at providing the DM a tool for him/her to learn about his/her preferences and their compatibility with the used preference model. In this sense, the presented empirical results are very promising in terms of applicability of the approach proposed in [11].

\section{Acknowledgements}

This work was partly supported by the LusoFrench Grant n328J4 (ICCTI/Ambassade de France).

\section{References}

[1] S.I. Gallant, Neural Network Learning, MIT Press, Cambridge, MA, 1993.

[2] J.W. Grzymala-Busse, LERS - a system for learning from examples based on rough sets, in: R. Slowinski (Ed.), Intelligent Decision Support: Handbook of Applications and Advances of the Rough Set Theory, Kluwer Academic Publishers, Dordrecht, 1992, pp. 3-18.

[3] E. Jacquet-Lagrèze, Interactive assessment of preferences using holistic judgments: The PREFCALC system, in: C. Bana e Costa (Ed.), Readings in Multiple Criteria Decision Aid, Springer, Berlin, 1990, pp. 335-350.

[4] E. Jacquet-Lagrèze, R. Meziani, R. Slowinski, MOLP with an interactive assessment of a piecewise-linear utility function, European Journal of Operational Research 31 (3) (1987) 350-357.

[5] E. Jacquet-Lagréze, J. Siskos, Assessing a set of additive utility functions for multicriteria decision-making, the UTA method, European Journal of Operational Research 10 (2) (1982) 151-164.

[6] J.G. March, Bounded rationality, ambiguity and the engineering of choice, in: D.E. Bell, H. Raiffa, A. Tversky (Eds.), Decision Making, Descriptive, Normative and Prescriptive Interactions, Cambridge University Press, New York, 1988, pp. 33-58.

[7] V. Mousseau, J. Figueira, J.Ph. Naux, Using assignment examples to infer weights for ELECTRE TRI method: Some experimental results, Cahiers du LAMSADE no 150, Université de Paris-Dauphine, 1997.

[8] R.S. Michalski, A theory and methodology of inductive learning, Artificial Intelligence 20 (1983) 11-116.

[9] V. Mousseau, Problèmes liés à l'évaluation de l'importance relative des critères en aide multicritère à la décision: Réflexions théoriques, expérimentations et implémentations informatiques, $\mathrm{PhD}$ thesis, Université Paris-Dauphine, 1993.

[10] V. Mousseau, Eliciting information concerning the relative importance of criteria, in: P. Pardalos, Y. Siskos, C. Zopounidis (Eds.), Advances in Multicriteria Analysis, Nonconvex Optimization and its Applications, Kluwer Academic Publishers, Dordrecht, 1995, pp. 17-43.

[11] V. Mousseau, R. Slowinski, Inferring an ELECTRE TRI model from assignment examples, Journal of Global Optimization 12 (2) (1998) 157-174.

[12] V. Mousseau, R. Slowinski, P. Zielniewicz, ELECTRE TRI 2.0a: Methodological guide and user's documentation, Document du LAMSADE no. 111, Université de ParisDauphine, 1999.

[13] V. Mousseau, R. Slowinski, P. Zielniewicz, A user-oriented implementation of the ELECTRE TRI method integrating preference elicitation support, Computers \& Operations Research (2000), to appear.

[14] J.-Ph. Naux, Calage d'un modèle electre tri à l'aide d'actions exemples, Mémoire de DEA, Université ParisDauphine, 1996. 
[15] R. Nadeau, J.-M. Martel, L. Kiss, ELECCALC: Modelling the decision maker's preferences with ELECTRE II, in: M. Cerny, D. Gluckaufova, D. Loula (Eds.), Multicriteria Decision Making: Methods, Algorithms and Applications, Czechoslovak Academy of Sciences, Prague, 1992, pp. 109-119.

[16] Z. Pawlak, R. Slowinski, Rough set approach to multiattribute decision analysis, European Journal of Operational Research 72 (1994) 443-459.

[17] J.R. Quinlan, Induction of decision trees, Machine Learning 1 (1986) 81-106.

[18] B. Roy, D. Bouyssou, Aide multicritère à la décision: Méthodes et cas, Economica, Paris, 1993.

[19] B. Roy, Main sources of inaccurate determination, uncertainty and imprecision in decision models, Mathematical and Computer Modelling 12 (10-11) (1989) 1245-1254.

[20] B. Roy, The outranking approach and the foundations of ELECTRE methods, Theory and Decision 31 (1) (1991) 49-73.

[21] B. Roy, Multicriteria Methodology for Decision Aiding. Nonconvex Optimization and its Applications, Kluwer Academic Publishers, Dordrecht, 1996.
[22] B. Roy, Ph. Vincke, Relational systems of preferences with one or more pseudo-criteria: Some new concepts and results, Management Science 30 (11) (1984) 1323 1334.

[23] R. Slowinski, Interactive multiobjective optimization based on ordinal regression, in: A. Lewandovski, V. Volkovich (Eds.), Multiobjective Problems of Mathematical Programming, LNEMS, vol. 351, Springer, Berlin, 1991, pp. 93-100.

[24] R. Slowinski, Intelligent Decision Support: Handbook of Applications and Advances of the Rough Set Theory, Kluwer Academic Publishers, Dordrecht, 1992.

[25] S.M. Weiss, C.A. Kulikowski, Computer Systems that Learn, Morgan Kaufmann, San Mateo, CA, 1991.

[26] W. Yu. Aide multicritère à la décision dans le cadre de la problématique du tri: Concepts, méthodes et applications, $\mathrm{PhD}$ thesis, Université Paris-Dauphine, 1992.

[27] W. Yu, ELECTRE TRI: Aspects méthodologiques et manuel d'utilisation, Document du lamsade no 74, Université Paris-Dauphine, 1992. 